\title{
LABORATORY OBSERVATIONS AND NUMERICAL MODELING OF THE EFFECTS OF AN ARRAY OF WAVE ENERGY CONVERTERS
}

\author{
Aaron Porter ${ }^{1}$, Merrick Haller ${ }^{1}$, and Pukha Lenee-Bluhm²
}

\begin{abstract}
This paper investigates the effects of wave energy converters (WECs) on water waves through the analysis of extensive laboratory experiments, as well as subsequent numerical simulations. Data for the analysis was collected during the WEC-Array Experiments performed at the O.H. Hinsdale Wave Research Laboratory at Oregon State University, in collaboration with Columbia Power Technologies, using five 1:33 scale point-absorbing WECs. The observed wave measurement and WEC performance data sets allowed for a direct computation of power removed from the wave field for a large suite of incident wave conditions and WEC array sizes. Using measured power absorption characteristics as a WEC parameterization for SWAN was developed. This parameterization was verified by comparison to the observational data set. Considering the complexity of the problem, the parameterization of WECs by only power absorption is a reasonable predictor of the effect of WECs on the far field. Keywords: waveenergy; spectral modeling; laboratory experiments
\end{abstract}

\section{INTRODUCTION}

\section{Background}

A clear understanding of how wave energy converters (WECs) will affect the ocean environment, ocean waves in particular, is needed before commercialization. In this paper, laboratory observations of the effects of WEC-arrays on the wave field are analyzed. In addition, a WEC parameterization for SWAN that is based solely on WEC power absorption is tested.

Wave energy converters currently have various designs, ranging from the point-absorbing buoy to on-shore installments, both of which generate power from the oscillating nature of ocean waves. In any case, momentum is transferred from the ocean waves to mechanical power, and in turn, electricity. WECs will also redistribute energy in addition to capturing energy. Power transfer from ocean waves to WECs, and the redistribution of wave power due to the presence of WECs create wave shadows shoreward of the device.

While test berths of WECs are expected to have few devices, and possibly only a single device, it is expected that WEC buoys will be deployed in arrays to employ efficiency in installment and maintenance. Hydrodynamic interactions between WECs may have constructive or destructive effects on the power removal from the superposition of multiple devices in an array. It is necessary to perform experiments that measure the effects of an array of WECs rather than solely a device in isolation.

There have been previous experiments, as well analytical and numerical models, which tested the effects of WEC-arrays. Experiments have been scarce, small in scope, and varied in the type of WEC used. Running WEC experiments is a difficult task in part because of small response signal (Boyle et al. 2007). Ashton et al. (2009) measured the effects of an array of a floating oscillating water column device on the surrounding wave field, but data analysis was limited to five sparsely dispersed wave gages. They found that single point measurements are not suitable to quantify the effect of a WEC on the surrounding wave field, but did not test the ability of wave gages placed in transects. Alexandre et al. (2009) ran physical experiments with $1 / 67$ th scale heaving point-absorbing WECs and tracked the changes made to the spectra in the physical experiment between incident and lee conditions. They found that group velocity had changed from the incident wave climate, as well as the spectral shape, which had become bi-modal when the WEC was tuned to the peak frequency of the incident spectra. Haller et al. (2007), using the present data set, found that the shadow was not dependent on incident wave height, but primarily upon wave period and array size.

Numerical modeling has been done with several methods, but none has yet been constrained by observational data sets. Much of the far-field modeling has been done with either spectral models (Millar et al. 2007; Smith et al. 2012; Silverthorne and Folley 2011) or with MildWAVE (Troch et al. 2011). Several papers have attempted to use a spectral model to predict the effects of WEC-arrays at the shoreline. Millar et al. (2007) and Smith et al. (2012) used SWAN to show that shoreline effects from a WEC-array four kilometers off the coast of Southwest England were minimal, but the results were unconstrained by empirical data. The MildWAVE model accounts for some WEC-WEC

\footnotetext{
${ }^{1}$ School of Civil and Construction Engineering, Oregon State University, 220 Owen Hall, Corvallis, Oregon, 97331, Benton County

${ }^{2}$ Columbia Power Technologies, 4920 SW $3^{\text {rd }}$ Street, Corvallis, Oregon, 97333, Benton County
} 
interaction, and Troch et al. (2011) concluded that staggered WEC-array arrangement resulted in more power absorption, but again the results were unconstrained by empirical data.

This paper will close the gap between numerical models and empirical data by parameterizing WEC effects by the absorption process only, running SWAN with this assumption, and comparing model results to data from the WEC-Array Experiments. Getting to this point required much data analysis, and parameterization of measured WEC effects upon the wave field. What follows is an explanation of experimental setup, the characterization and parameterization of the wave shadow due WEC-arrays, implementation of a numerical model based on observational data, and the validation of such a model.

\section{WEC-ARRAY EXPERIMENTS}

\section{Experimental Setup}

Experiments were performed at the O.H. Hinsdale Wave Research Laboratory in the Tsunami Wave Basin during the winter of 2010/2011. These experiments, known as the WEC-Array Experiments, were performed in conjunction with Columbia Power Technologies (CPT). CPT provided five 1:33 scale WECs for use in the experiments; the "Manta 3.1", a point-absorbing WEC that generates power in both heave and surge motions. The Manta has both an aft and fore float, which are attached to a heavy spar through a drive shaft, as shown in Figure 1.

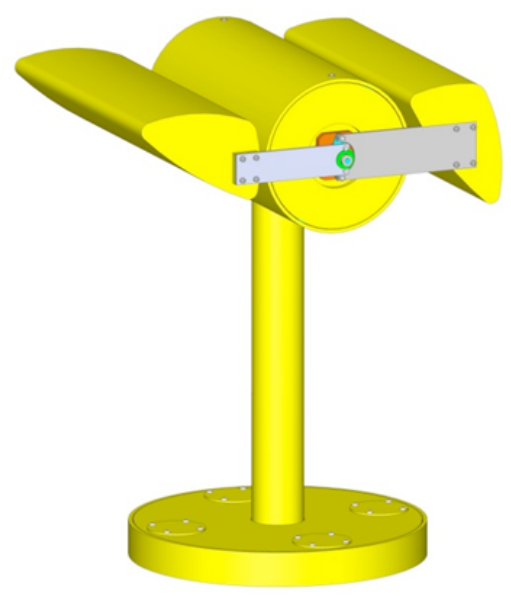

Figure 1. Columbia Power Technologies'“Manta 3.1" wave energy converter

As incoming waves pass the WEC, heave and surge motions force the floats to rotate about the top portion of the spar and drive the direct drive rotary (DDR) generators. At lab scale the devices have a diameter of 0.55 meters and in the field this scales to approximately eighteen meters. In the water at any one time were one, three, or five devices, with like wave conditions run for each array size. WEC array positions are illustrated in Figure 2.

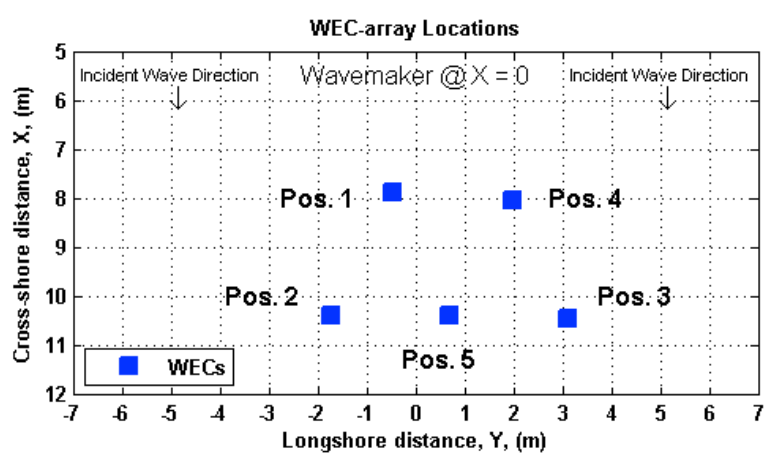

Figure 2. WEC positions in the Tsunami Wave Basin 
The instrumentation for these experiments included twenty-three wave gages, an ultrasonic wave gage (USWG), and three acoustic doppler velocimeters (ADVs). Wave gages were placed in arrays designed to measure and resolve directionally spread incident wave fields, lee wave fields, wave scattering, far-field effects, and any cross-shore reflections. Wave gage setup in the basin is shown in Figure 3, and shows the offshore gage array, which measured the incident wave conditions. The six gages directly in the lee of the array (lee gage array) measured the affected waves.

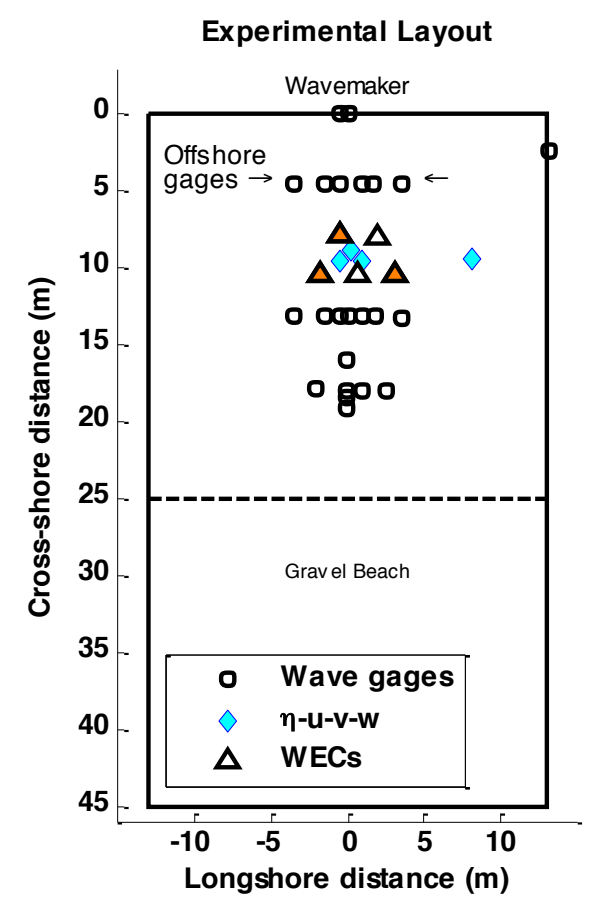

Figure 3. Experimental Layout of The WEC-Array Experiments. Black circles are wave gages, triangles are WECs, with orange triangles representing the WEC arrangement in a three device array, and the blue diamonds are co-located wave gage and ADVs.

\section{Wave Conditions}

Wave conditions in the WEC-Array Experiments consisted of regular waves and parameterized real seas sea states. Regular waves were tested with a suite of incident wave periods and wave heights, and from two directions: head on, and off angle at $22.5^{\circ}$. In this paper, normally incident waves with a target height of six centimeters were considered.

\section{DATA ANALYSIS}

\section{Preliminary Results}

Wave gage measurements from the lee array are used to quantify the power deficit induced by the WEC array. For the purposes of this paper, wave heights recorded at the lee gage array were analyzed from select wave cases only. It had been shown previously in work with this data set that the wave shadow behaves linearly with increased incident wave heights (Haller et al. 2011).

It was clear that the decrease in wave height observed at the lee gage array depends on WEC array size. Larger WEC-arrays induce a deeper shadow, and the wider WEC arrays (the three and five device arrays) induce wider shadows. Our previous work on the WEC-Array Experiments data set showed that the relative wave height reduction (or wave shadow), that is the percent change from incident wave heights at the lee, increases as the wave period becomes shorter (Haller et al. 2011).

In this paper the incident wave conditions have been specified as the wave characteristics measured at gages one through six (the offshore gage array) and gage ten in trials where a single WEC was present only. Trials with like target wave conditions for three and five WEC arrays also have their incident wave measurements specified from the incident measurements of the single WEC case. This 
decision was made after much work determining that the wave heights measured at the offshore gages are increased by the presence of larger WEC arrays.

The magnitude of the wave shadow due to the WECs was characterized by measuring the amount of power "lost" from the wave field between the incident and lee gage array. Wave power along the lee gage array was determined by a spatial integration of the shoreward energy flux at each of the gages. Incident power was specified as the average incident energy flux for each unique wave conditions case, and then multiplied by the length of the lee gage array. Average incident energy flux is the averaged measured energy flux from gages one through six and ten, for each wave condition. Energy flux at each gage for a regular wave is given by:

$$
E f=\frac{1}{8} \rho g H^{2} \frac{L}{T} * \frac{1}{2}\left(1+\frac{2 k h}{\sinh (2 k h)}\right)=E C n=E C_{g}
$$

In this equation, $\rho$ is density of water, $g$ is gravity, $H$ is wave height, $L$ is wave length, $T$ is wave period, $k$ is wavenumber, $h$ is depth, $E$ is energy, $C$ is wave celerity, and $C g$ is group velocity. In spectral seas (real sea) energy flux at each gage is defined as:

$$
E f=\int \rho g \cdot C_{g} \cdot S_{i} \cdot d f
$$

where $S$ is the spectral density at each frequency. Power is then the spatial integration of energy flux in the alongshore direction of the gage arrays:

$$
P_{\text {gageArray }}=\int E f_{i} \cdot d y
$$

For regular wave trials of one, three, and five WEC-arrays, the incident power and lee gage array power was compared and the losses between the two sets calculated. Figure 4 shows power loss (deficit) measured in the wave field for a range of regular wave periods only, with the three array sizes.

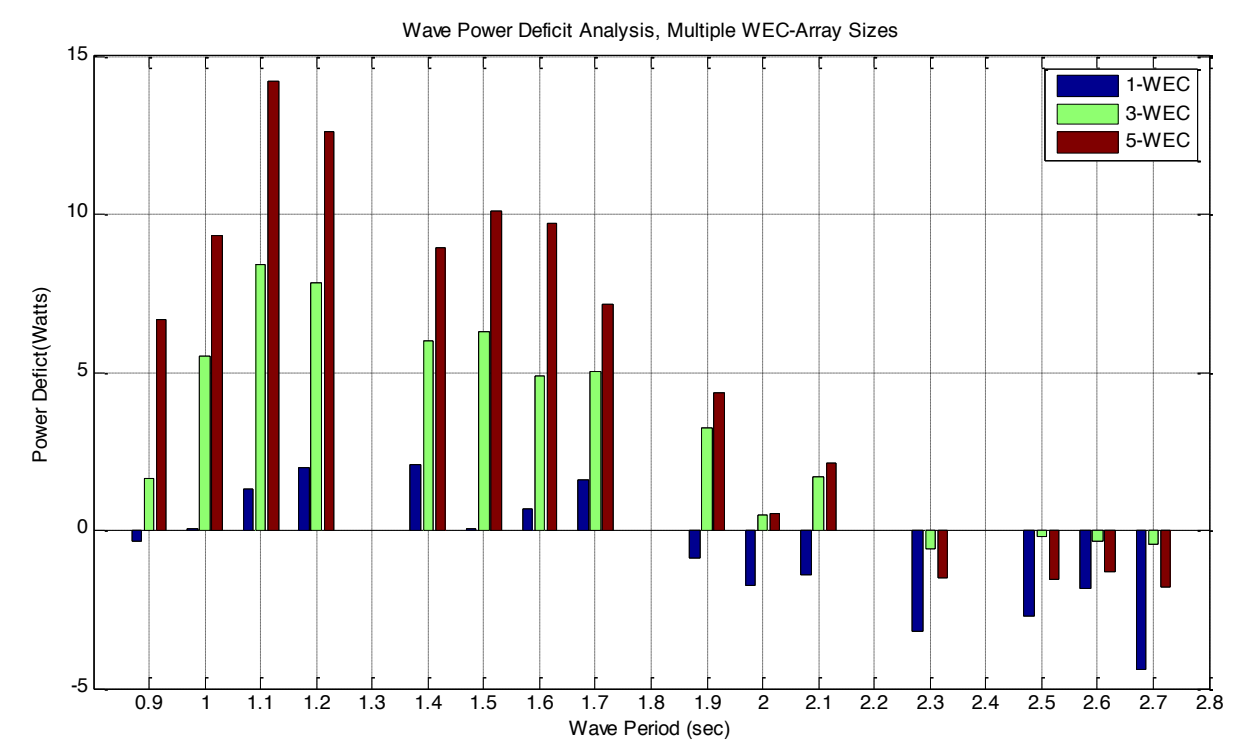

Figure 4. Wave power deficits measured between incident wave conditions and the lee gages for regular wave trials. Power was measured as the longshore integration of energy flux. Negative values occur in high periods due to very low signal-to-noise ratios.

There is a clear pattern to the power deficits between incident and lee power measurements, with peak power loss occurring at 1.2 seconds, and then gradually decreasing on either side. At high period waves an apparent power gain is visible, which is the result of low signal to noise ratios due to very high incident wave power, and very low WEC absorption at those periods. Power deficits are largest when the 5-WEC array is in the water, and smallest with the 1-WEC array. Of interest is to what extent the wave power lost scales between array sizes. 


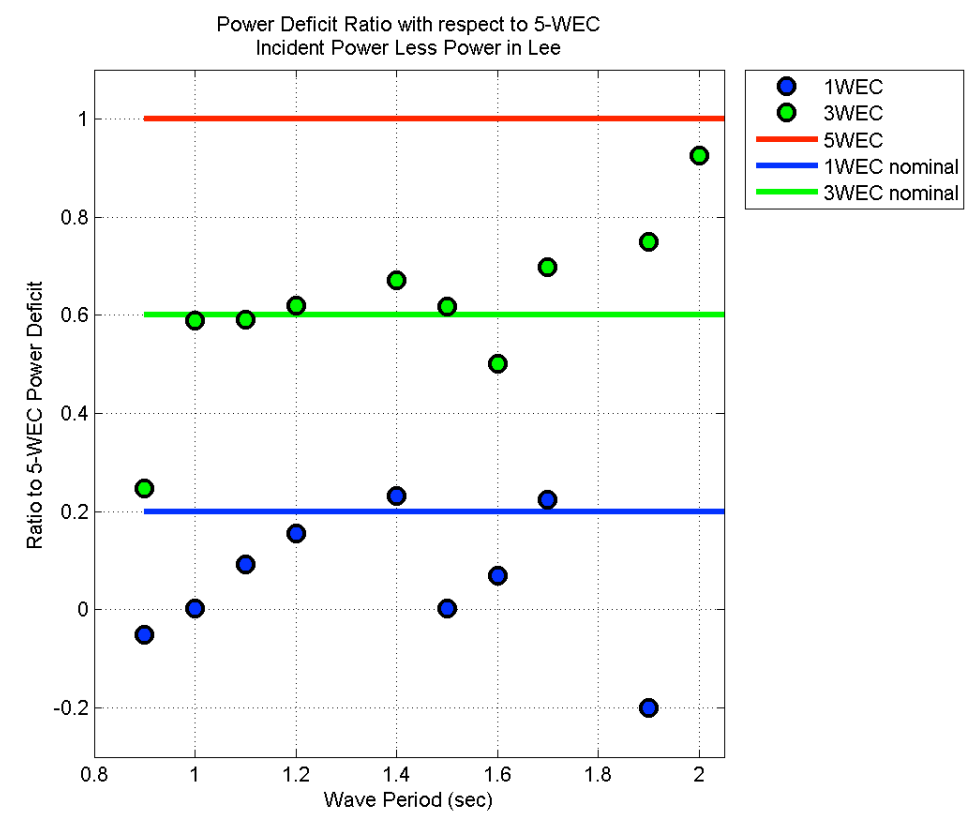

Figure 5. Ratios of power deficits in regular waves between differently sized arrays, with respect to the 5WEC array. The green dots represent the ratio of power deficits measured in a suite of wave periods between the 3-WEC arrangements and the 5-WEC arrangements, which shows moderate linearity since they plot close to the green line. Blue dots (1-WEC to 5-WEC) are more variable due to low signal-to-noise ratios.

Each of the array sizes was subject to different amounts incident wave power and net power capture depends on the array size since there were more or less WECs in the water. Figure 5 shows the power deficit scaling between array sizes, which is moderately linear. The lines represent what would be a perfectly linear system, where the power deficits of three and one device arrays would be exactly three fifths and one fifth of the power deficit in a five-device array, respectively. The normalized power deficit with respect to available power is the ratio of power deficit and the sum of the wave power available to the WECs. The Wave Power Available (WPA) is defined as:

$$
W P A=W \cdot E f \cdot \# W E C S
$$

Where, $W$ is the width of each WEC $(0.55 \mathrm{~m}), E f$ is average incident energy flux (Watts $/ \mathrm{m}$ ), and $\#$ WECS is the number of devices in each WEC array $(1,3$ or 5$)$.

Of interest is the ratio of power takeoff to WPA. Power takeoff from the Manta was determined using the optical motion tracking system, which enabled the calculation of velocity, $\omega$, torque, $\tau$, and power, $P$ for each wave condition. Performance of the device was characterized by relative capture width $(\mathrm{RCW})$ :

$$
R C W=P / W P A
$$

To compare the mechanical power absorption efficiency to wave data characteristics we compared $\mathrm{RCW}$ to the measured power removed in the waves between the incident and lee measurements relative to the incident wave power. We call this the Relative Influence Width (RIW), defined as:

$$
R I W=P_{\text {Deficit }} / W P A
$$

where power, $P$, deficit is the measured power deficit between the incident and lee gages.

Figure 6 plots the RIW values for regular waves in one (blue), three (green) and five (red) device arrays, as well as the RCW (in black). The three and five device RIW curves display similar effective relative influences on the incident wave power, compared to the single device array, because the shadow signals from larger arrays are better resolved by the wave gage measurements. For small wave periods, the differences between RIW and RCW are greater, indicating some other process than absorption is likely occurring. However, across most periods it was clear that the RIW and RCW follow a similar trend to the RCW curve. This suggests a model WEC parameterization might be 
developed based on absorption characteristics only. Such a parameterization of WEC effects was tested with the spectral model SWAN.

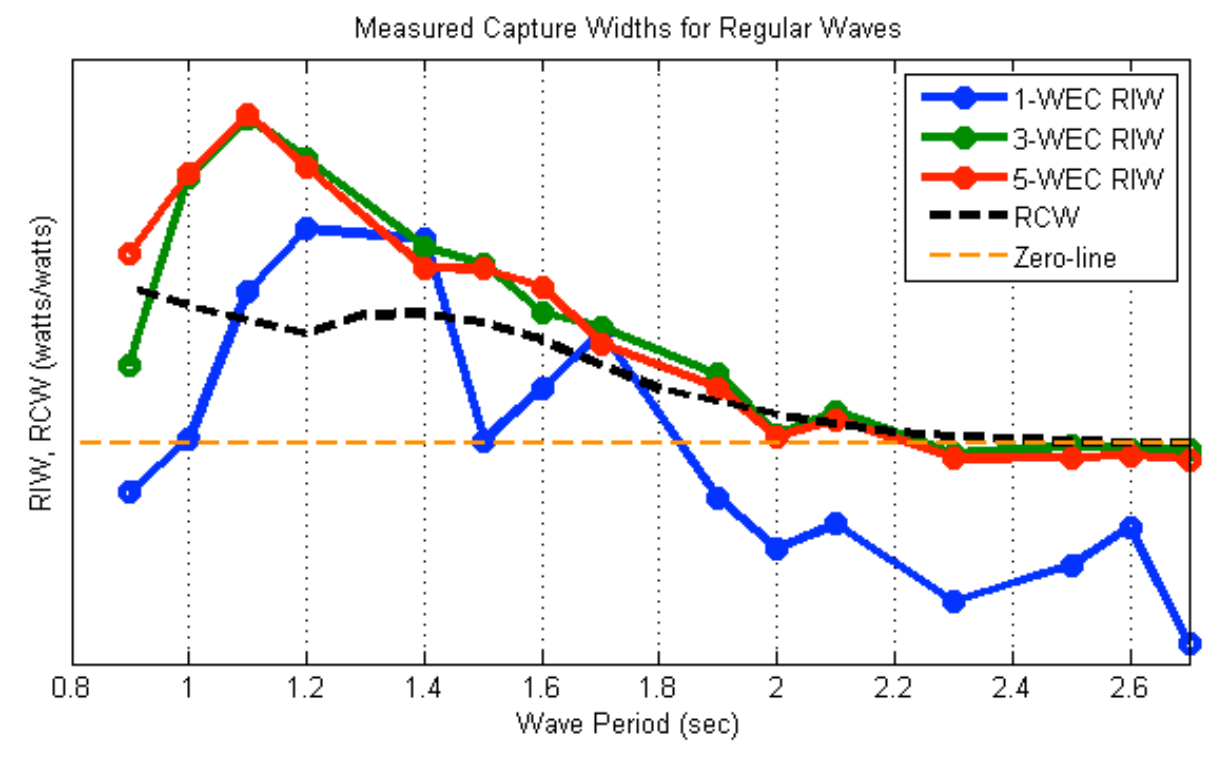

Figure 6. Relative power capture widths of mechanical power absorbed, and power deficits in the wave field of regular wave trials. Colored lines represent the wave power deficits measured between the incident wave field and lee gage array wave field. The black line represents relative power captured by the WEC. The orange line would indicate no change from the incident wave conditions.

\section{WEC EFFECT PARAMETERIZATION AND MODELING}

\section{NUMERICAL MODELS}

Because the mechanically calculated RCW curve is reasonable similar to the RIW measured from the wave data, we can try and predict the changes to the wave field based solely on the device absorption behavior. In this parameterization scattering of waves due to the presence of the WECs was not considered.

\section{SWAN Background}

In this analysis the spectral wave model SWAN was used. SWAN is a third generation spectral wave model that models the wave action density balance. The model numerically represents the effects of spatial propagation, refraction, shoaling, wave generation, dissipation, and nonlinear wave-wave interactions over real bathymetry (Booij et al., 1999). The bathymetry was measured from a LIDAR survey of the tsunami wave basin, performed by Dr. Michael Olsen and the OSU Geomatics unit. The wave action balance equation reads:

$$
\frac{\partial N}{\partial t}+\frac{\partial c_{x} N}{\partial x}+\frac{\partial c_{y} N}{\partial y}+\frac{\partial c_{\sigma} N}{\partial \sigma}+\frac{\partial c_{\theta} N}{\partial \theta}=\frac{S_{t o t}}{\sigma}
$$

where $\sigma$ is radial frequency, $N$ is the energy density $E(\sigma, \theta)$ distributed over radian frequencies, and propagation directions, $\theta$. On the right side of the equation $S_{\text {tot }}$ is the sum of the sources and sinks that generate, dissipate, or redistribute wave energy. This analysis does not account for the lateral redistribution of wave energy, as the diffraction approximation in SWAN is sensitive to the necessary mesh size to wavelength ratio. Additionally there was no wave generation due to wind and the only losses in $S_{t o t}$ were due to wave breaking. Typically, an obstacle that removes power from the wave field is included in the sink term in $S_{\text {tot }}$; however, the most current SWAN code does not allow for frequency dependent wave power absorption (sinks). We know that WECs remove power on a frequency dependent basis, so a modification external of the SWAN program was needed to properly resolve the WEC-array effects. 


\section{Parameterization of WEC Effects}

The effects of the WEC-arrays are parameterized similarly for regular waves and real seas. In both cases the percent of power passing at each frequency in the incident power spectrum is determined by the RCW curve value, with the transfer function:

$$
\text { TransferFunc }_{i}=1-R C W_{i}
$$

This transfer function is multiplied by the incident wave spectra at each frequency to represent WECeffects:

$$
\text { WECspec }=\text { IncSpec } \cdot \text { TransferFunc }
$$

At each grid point in the longshore domain where a WEC is present, the transfer function is applied externally, outside of the SWAN program. In the case where there are multiple rows of WECs in the array, this method is applied multiple times in a step-wise fashion. For the case of a 5-WEC array, shown in Figure 7, SWAN is split into three nests. Nest 1 models just the incident wave field with its shoreward cross-shore boundary as the longshore transect of the first row of WECs. Spectra at each WEC grid point are modified by the transfer function with the rest of the points unchanged. These spectra along the entire longshore boundary of Nest 1 are the incident spectra for Nest 2 and the process is repeated for the subsequent rows of WECs. SWAN was run separately for each nest.

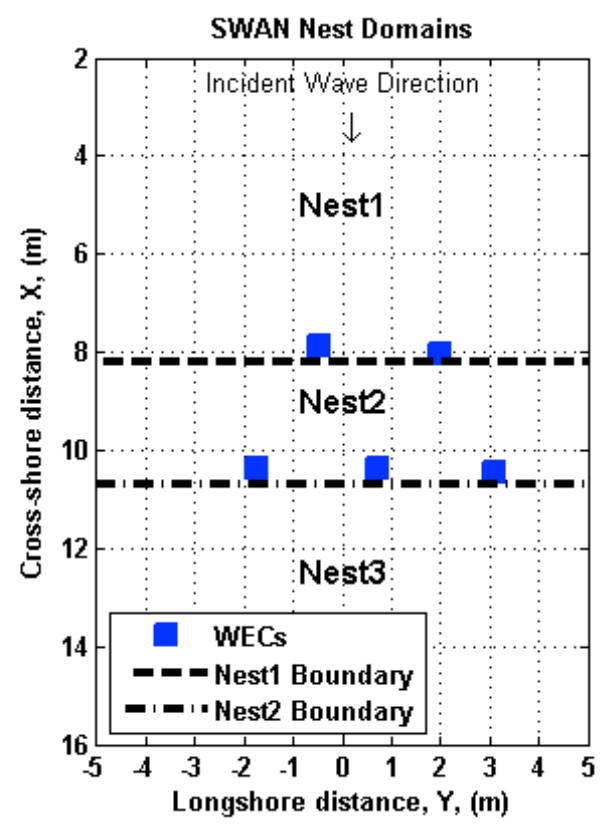

Figure 7. Nests used in the SWAN domain with locations of WECs pictured in blue. Black dashed lines are the nest boundaries.

\section{Model Results}

Previously in this paper, we measured the influence of WEC-arrays on the waves by the power deficit observed in the lee gage array relative to the incident wave conditions. The same method was applied to model results. Power deficits measured in the wave data from the WEC-Array Experiments were compared to deficits calculated from the model results for the same incident conditions. Figure 8 shows the model to data comparison for a 5-WEC array in regular waves, where data is in black and model results are in copper. Only results from the 5-WEC array conditions were shown since the WECarray shadow signal was strongest at this size. At most wave periods the model resolves power deficits reasonably well, but in several of the shorter wave periods (1.0-1.2 seconds) much larger power deficits were measured in the wave data than predicted by the mode. This was not surprising; as we saw in the $\mathrm{RCW} / \mathrm{RIW}$ comparison the effective width measured in the wave data was much larger than predicted than the RCW curve (mechanical absorption) at the same regular wave periods (1.0-1.2 seconds). 


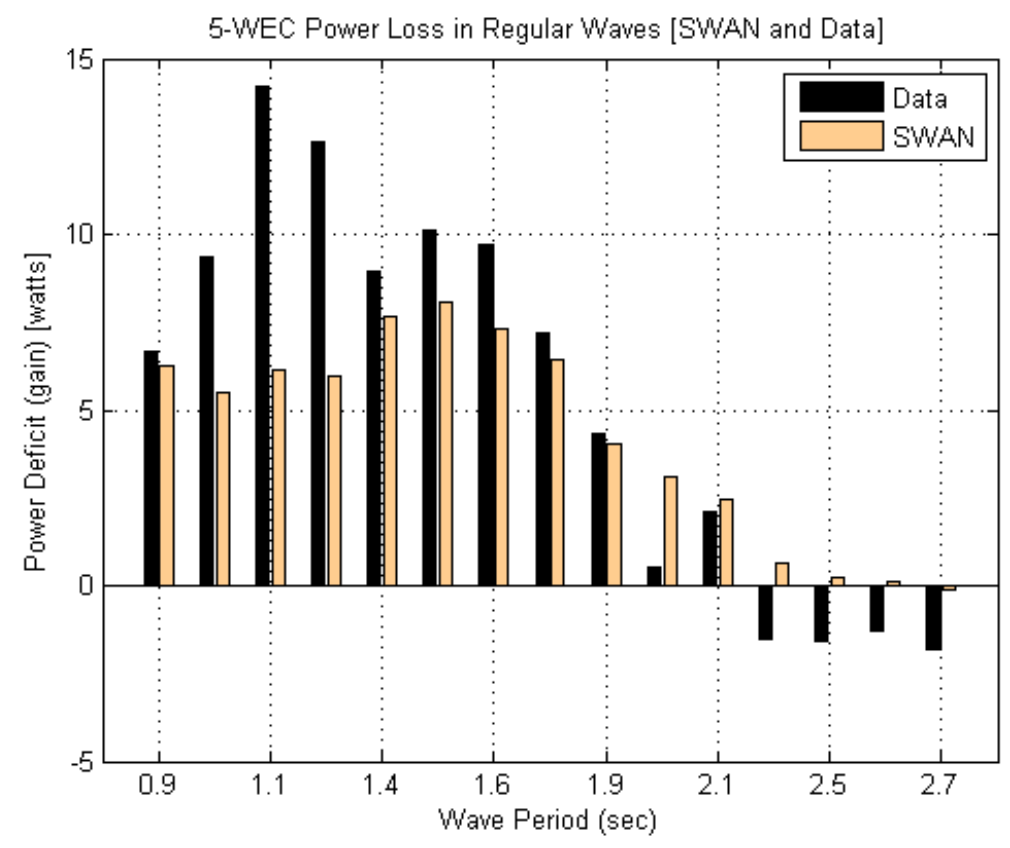

Figure 8. Comparison of model results (copper) to empirical data (black) in regular waves for a 5-WEC array.

\section{CONCLUSIONS}

A reasonable representation of the effects of an array of wave energy converters on regular waves was achieved by the parameterization of WECs based on the absorption process only. The observational data set from the WEC-Array Experiments was an excellent resource for investigating WEC effects on the wave field, and for model verification. Data and results from trials run with real seas conditions are currently being computed and so far look promising. Application of this method to field scale predictions in real seas conditions is the final goal.

\section{ACKNOWLEDGMENTS}

This research was supported by the U.S. Department of Energy (DE-EE0002658), Sandia National Labs, and Columbia Power Technologies under Research Subagreement NO. 2010-1698, additional support came from Oregon Wave Energy Trust through Award Number OIC-0911-109. We would also wish to thank the staff of the Hinsdale Wave Research Laboratory for their considerable effort.

\section{REFERENCES}

Alexandre, A., Stallard, T., Stansby, P.K. 2009. Transformation of Wave Spectra across a Line of Wave Devices. Proceedings of the 8th European Wave and Tidal Energy Conference (EWTEC).

Booij, N., Ris, R. C., Holthuijsen, L. H. 1999. A third generation wave model for coastal regions 1. Model description and validation, Journal of Geophysical Research, 104, 7649-7666

Boyle, L., Elsaesser, B., Folley, M., Whittaker, T. 2011. Assesment of Wave Basin Homogenuity for Wave Energy Converter Array Studies. Proceedings of the 9th European Wave and Tidal Energy Conference (EWTEC).

Haller, M., Porter, A., Lenee-Bluhm, P., Rhinefrank, K., Hammagren, E., Ozkan-Haller, H. T., and Newborn, D., 2011. Laboratory observations of waves in the vicinity of WEC-arrays. Proceedings of the 9th European Wave and Tidal Energy Conference (EWTEC).

Millar, D.L., Smith, H.C.M., Reeve, D.E. 2007, Modelling analysis of the sensitivity of shoreline change to a wave farm. Ocean Engineering, 34, 884-901.

Silverthorne, K., Folley, M. 2011. A new Numerical Representation of Wave Energy Converters in a Spectral Wave Model. Proceedings of the 9th European Wave and Tidal Energy Conference (EWTEC).

Smith, H. C. M., Pearce, C., Millar D. L. 2012. Further analysis of change in nearshore wave climate due to an offshore wave farm: An enhanced case study for the proposed Wave Hub site. Renewable Energy, 40, 51-64. 